Projets

de paysage

\section{Projets de paysage}

Revue scientifique sur la conception et l'aménagement de l'espace

\section{$7 \mid 2012$}

Réédition d'articles du séminaire «Étapes de recherches en paysage » 2000-2006

\title{
Les anciens vignobles « ornements des villes » : legs, images, opportunités, perspectives
}

Étude de cas appliquée à l'agglomération de Nancy

Ancient vineyards as ornaments of the city: legacies, perception, and prospects A case study applied to the metropolitan area of Nancy

Jean-Pierre Husson

\section{(2) OpenEdition}

\section{Journals}

\section{Édition électronique}

URL : https://journals.openedition.org/paysage/16498

DOI : $10.4000 /$ paysage. 16498

ISSN : 1969-6124

\section{Éditeur :}

École nationale supérieure du paysage de Versailles-Marseille, Institut national des sciences appliquées Centre Val de Loire - École de la nature et du paysage, École nationale supérieure d'architecture et de paysage de Bordeaux, École nationale supérieure d'architecture et de paysage de Lille, Agrocampus Angers

\section{Référence électronique}

Jean-Pierre Husson, «Les anciens vignobles « ornements des villes » : legs, images, opportunités, perspectives », Projets de paysage [En ligne], 7 | 2012, mis en ligne le 04 janvier 2012, consulté le 08 mai 2021. URL : http://journals.openedition.org/paysage/16498 ; DOI : https://doi.org/10.4000/ paysage. 16498

Ce document a été généré automatiquement le 8 mai 2021.

\section{(c) $)(9)$}

La revue Projets de paysage est mise à disposition selon les termes de la Licence Creative Commons Attribution - Pas d'Utilisation Commerciale - Pas de Modification 4.0 International. 


\title{
Les anciens vignobles « ornements des villes » : legs, images, opportunités, perspectives
}

\author{
Étude de cas appliquée à l'agglomération de Nancy
}

Ancient vineyards as ornaments of the city: legacies, perception, and prospects A case study applied to the metropolitan area of Nancy

\section{Jean-Pierre Husson}

La ville ancienne (Lepetit, 1980), close ou médiocrement étalée en dehors de ses murs, était prolongée par des faubourgs digités. Elle était entourée de ceintures nourricières indispensables à son fonctionnement afin de produire les subsistances qui circulaient peu ou mal afin d'assurer ses approvisionnements vitaux. Il s'agissait surtout de jardins, de vergers, d'étangs souvent associés à des héritages de défense, utilisés pour produire des poissons et également fournir la force motrice nécessaire aux moulins. Des prairies occupaient encore les délaissés, en particulier les zones d'étale du lit majeur du fleuve voisin longtemps redouté avant qu'il ne fût cadenassé et, par là, conduit dans une suite de dysfonctionnements ultérieurs. Cet environnement comptait encore des garennes pour satisfaire le plaisir du prince. Des résidences d'été ceintes de murs, surlignées par des bois marmenteaux et des fondations pieuses, s'accrochaient aux versants les mieux exposés des campagnes les plus proches. Les nombreuses vues cavalières puis les plans qui s'égrènent du XVIe au XVIII ${ }^{e}$ siècle font le constat de cette situation mise en scène. À échelle fine, les cartes dites topographiques, les plans utilisés comme titres de propriété puis les matrices cadastrales anciennes montrent des parcellaires fonciers très émiettés parfois transmis jusqu'à nous. Dans cet environnement de nature qui s'immisce et résiste, la vigne est l'ornement (Dion, 1990) ordinaire des cités qui sont rarement synchrones avec elles-mêmes et subissent des décalages d'occupation des sols (Roncayolo, 1996). La vigne joue alors un rôle essentiel dans l'organisation des mosaïques paysagères, en particulier là où elle exige du zèle pour soumettre des lieux indociles (Roupnel, 1955). Cette culture noble, monastique ou paysanne, s'effectue essentiellement à la main. Ad minima, elle mobilise peu d'outil et de 
capitaux, sauf s'il s'agit de clos. Avec la pioche, le pic, des outils pour tailler les ceps et surtout du travail et des soins, on peut être récompensé de son labeur si les conditions climatiques sont de la partie. La vigne produit une boisson indispensable et faite pour la socialisation: libations pour le plaisir, vins de messe, parfois ivresse des villes de garnison ou de foires, mais également breuvage coupé pour servir à purifier l'eau de source trop parcimonieusement distribuée dans la citée et remplacée par celle des puits souvent pollués par les effluents. Le legs de cet héritage associé à une ville qui se construit et se refait sur elle-même est important. Il est formé de tiers espace naturel, nourricier et qui représente maintenant un fort potentiel de biodiversité ordinaire (Godet, 2010).

2 L'exposé part d'un inventaire du legs reçu, imprimé dans la matrice foncière et la microtopographie urbaine commence à être identifiée par le balayage LIDAR $^{1}$ exhumant les éléments fossilisés dans l'inertie des formes d'occupation de l'espace. La vigne, décor de la ville, a été successivement agrandie, dégradée, remplacée, gommée avant d'être aujourd'hui récupérée, partiellement restaurée. Le préalable de reconnaissance est à associer à nos actuelles envies de villes. Celles-ci sont largement formatées par la transition en cours entre l'application parfois sans nuance des préceptes de la charte d'Athènes en direction de ceux mis en avant par celle d'Aalborg. Désormais, nous réfléchissons à mieux intégrer et préserver les espaces flous, délaissés, de nature ordinaire qui donnent aux agglomérations une certaine capacité à respirer et à abriter une abondante biodiversité ordinaire parfois menacée par les invasives. Cet enjeu repose sur la capacité à articuler ensemble les outils de décision dessinés dans des échelles complémentaires afin de faire fonctionner le système "citadinisé » et, si possible, lui apporter un supplément d'aménité et de convivialité. La renaissance voulue passe par l'orchestration d'événements, de moments festifs appuyés sur une bonne communication à propos de ces espaces verts atypiques. La démonstration s'appuie sur l'étude de cas de l'agglomération de Nancy.

\section{Le legs viticole dans des villes qui s'empilent}

3 La présence de la vigne s'inscrit dans l'analyse spatiale de la ville. L'inertie de sa présence a eu tendance à bloquer et à arrêter la production d'espace urbain qui n'a jamais été isotrope (Arnaud, 2008). Elle a maintenu des parcellaires très éclatés, peu compatibles avec le changement d'affectation des sols. La présence passée de la vigne invite à réfléchir à l'échelle de l'îlot, du groupe de parcelles réduites, hachées par les partages et les héritages. Ces divisions sont compréhensibles quand elles sont rapprochées de l'investissement en travail accumulé. La valeur des terres explique les partages presque à l'infini des pièces travaillées. La vigne a eu tendance à investir les moindres arpents, les terres pentues, caillouteuses, là où d'autres pratiques eussent été impensables. Quand de longues périodes de paix se dessinent, et suite aux démembrements des fortifications médiévales, des douves, escarpes et contre-escarpes, les vignes sont plantées partout. En 1722, le parlement de Metz doit ordonner l'arrachage des vignes car cette cité épiscopale encore enclavée dans les duchés conserve un rôle stratégique de premier plan qui impose de disposer d'espaces environnants ouverts. Après 1756, l'essor de la vigne périurbaine se généralise, l'interdiction de planter ayant été levée à l'échelle du royaume. Les villes sont alors assaillies par un écrin de jardins et de treilles (4 200 ha autour de Paris dès la fin du 
$\mathrm{XVIII}^{\mathrm{e}}$ siècle). Au XIX ${ }^{\mathrm{e}}$ siècle, cette vigne revêt souvent une dimension festive associée au caractère agreste des lieux. L'exemple montmartois reste le plus connu, avec des lieux qui furent chantés (Aristide Bruant), peints (Suzanne Valadon, Maurice Utrillo, Francisque Poulbot, etc.), entrés dans l'Histoire (la Commune de Paris). Avec les jardins avec qui elle s'apparente dans la façon de mobiliser les efforts, la vigne, qui réclame moins que le potager la tyrannie de la présence de l'eau, peut s'accommoder de presque tous les terroirs. Cependant, elle préfère les bonnes expositions ensoleillées et, si possible, recherche la proximité des lisières protectrices si l'on se situe dans des territoires à climat teinté de semi-continentalité. Le rideau boisé atténue alors les chocs thermiques. Les lisières héliophiles favorisent la croissance du robinier couramment utilisé pour tailler des échalas. Dès le XIX ${ }^{\mathrm{e}}$ siècle, le faux acacia détrône souvent la place occupée par le châtaignier. La culture ancienne des ceps autorise de fortes densités. Le provignage et le complantage cumulent leurs effets pour optimiser l'occupation du sol. Le premier, qui consiste à coucher dans une fosse le corps du cep, enlève toute géométrie à la culture; le second crée une organisation étagée où l'arbre et les banquettes de terre jardinées peuvent cohabiter au sein de très petites parcelles. Chacune doit être accessible et permettre l'évacuation du produit des vendanges. Il en ressort l'existence et le maintien d'un lacis de chemins souvent fort étroits, parfois privatifs s'ils sont dessinés à la limite de deux parcelles. Ils sont alors identifiés par l'usage mais seulement surlignés sur les matrices cadastrales. Cette densité des chemins tend à brouiller l'intelligibilité des formes d'occupation des sols, avec l'alternance, voire l'intrication de mosaïques complexes et résistantes, de zones construites et encore de vastes espaces, domaines nobles ou religieux organisés d'un seul tenant. À Nancy, tout est drapé de vignobles agencés en minuscules parcelles, avec dégradation en lambeaux dès que la pente disparaît. Dès lors s'impose la présence des grandes propriétés couvrant généralement de 80 à 120 ha, souvent d'un seul tenant (Prêcheur, 1953), bornant l'espace non construit et en dessinant les verrous. Ainsi, le comte Molitor est propriétaire de 374 ha sur Tomblaine. Ces possessions souvent dominées par un château ou une belle résidence d'été sont parfois ceints de murs, avec des bois puis des terres labourées, des près, des friches; bref la formation d'un géosystème cohérent qui, tout à la fois, produit et affiche le prestige du propriétaire.

4 La vigne a tissé de vieilles liaisons (Plet, 2002) avec la ville. La proximité de cette dernière a tendance à améliorer la qualité du produit, la rusticité étant alors plutôt, et sans généralisation hâtive possible, affaire de ruralité. La vigne, tour à tour, dilatée puis rétractée, menacée, mise en concurrence quand le chemin de fer facilite les échanges commerciaux puis quand advinrent les crises qui finirent par la détruire. Ainsi, sur le bassin de Neuves-Maisons, le déclin accéléré de la viticulture est presque contemporain de l'essor sidérurgique, un système économique balayant le précédent seulement attesté dans la morphologie des maisons vigneronnes fort différentes des maisons jointives associées au système de l'openfield. De cet épisode, il demeure aussi des palimpsestes agraires, une approche initialement conduite par Gaston Roupnel dans le regard qu'il portait sur les paysages agraires : «La vigne a été alternativement cette nouveauté qui pare le paysage et cette ruine qui l'altère.» $(1955$, p. 169.) Bref, la place de la vigne dans le dévidement du fil d'Ariane paysager qui rejoint les passés successifs à l'actuel invite à associer les temporalités, les espaces et les formes dessinées puis souvent figées des parcellaires très éclatés. Après avoir été oubliés, ces lieux reprennent du sens et de l'intérêt, avec la capacité de résilience (principe de stabilité dans l'évolution jusqu'à ce qu'une rupture ou bifurcation s'impose) qu'ils offrent par le 
rôle exercé comme objet de connexité. Les objets qui attestent de l'ancienne culture de la vigne apportent aussi un supplément de sens, voire de saveurs, aux lieux qui ont conservé des traces du passé. C'est par exemple les grosses pierres plates posées de champ, perpendiculairement à la pente afin de retenir la terre. On en retrouve partout quand on dresse un inventaire attentif, parcelle par parcelle là où il y a eu de la vigne. Elles sont souvent cachées par la friche ou encore conservées dans la mémoire du sol. Les espaces évoqués sont très souvent en déshérence, effondrés, plus rarement réappropriés. Ils ont évolué selon un enchaînement de causes, processus, voire blocages, avec désormais des liaisons fortes faites dans les trajectoires tissées entre écologie et histoire du paysage (Girel, 2006).

\section{Des objets oubliés et enfouis à la renaissance des lieux}

5 L'étalement urbain s'est d'abord effectué là où la mobilisation foncière était la plus aisée, sur les espaces peu accidentés et peu grevés de handicaps (mémoire des risques d'inondation, sols mal stabilisés, présence de cavités ou d'argiles soumises à de sévères processus de rétractation, etc.). Dans ce contexte, les parcellaires très éclatés ont résisté à l'étalement urbain et ont pu être occlus par celui-ci. La mobilisation foncière étant trop délicate à réussir, l'enclavement des parcelles était général. Ces espaces restés en survivance, presque gommés, à l'avenir a priori incertain, ont évolué dans l'oubli, avec transmission de la forme mais vidage du contenu (Robert, 2003) par disparition de la vigne. Cette évolution s'est bien souvent faite dans l'indifférence, voire parfois la mise en marge des lieux. In fine, après une longue léthargie et des décalages croissants entre l'existant et les raisons initiales de l'agencement des lieux, ceux-ci sont réintégrés dans des logiques urbaines pour les capacités d'aménité offertes. Ils s'insèrent désormais dans des logiques de convivance (Velascot-Graciet, 2009), de retour à une certaine harmonie entre l'espace et les attentes sociales. Ce passage traduit un déblocage de situation. Le contexte change. Dès lors s'imposent de nouvelles échelles d'approches des territoires, celles des espaces de l'écologie du paysage: la mosaïque, l'îlot boisé ou envahi par la friche, le linéaire connexe. À ce propos, Claude et Georges Bertrand avaient parlé de géographie traversière dans la mesure où s'envisage du renouveau en croisant temporalités et territoires mais aussi usages, mises en scène, fêtes et mises en ambiance. Bref, les espaces évoqués laissés dans l'oubli sont à nouveau éclairés par des projets qui les intègrent, des acteurs qui mesurent à nouveau tout le potentiel qu'ils peuvent offrir, y compris en faisant revivre la richesse évocatrice des toponymes conservés afin d'apporter une note sensible aux espaces réinvestis.

La renaissance est à la croisée de plusieurs paramètres. Il s'agit de l'envie associée à l'effet de mode dont peut profiter un territoire. Elle concerne également les outils mis en place par les aménageurs. Elle traduit surtout la volonté politique exprimée en tenant compte de la base, en particulier du travail des associations dont il faut cependant parfois canaliser les dérives, en l'occurrence ici les effets "Nimby ». Cette convergence autour de trois types d'actions forme une sorte de levain, d'alchimie à la fois fragile et vivifiante. Elle s'exprime dans la conduite d'un projet partagé. Il traduit trois volontés : la promotion de l'existant et son préalable d'inventaire afin de réussir un zonage, la promotion par le désenclavement puis l'affichage du territoire concerné à mettre en scène, à faire connaître par une action événementielle. 


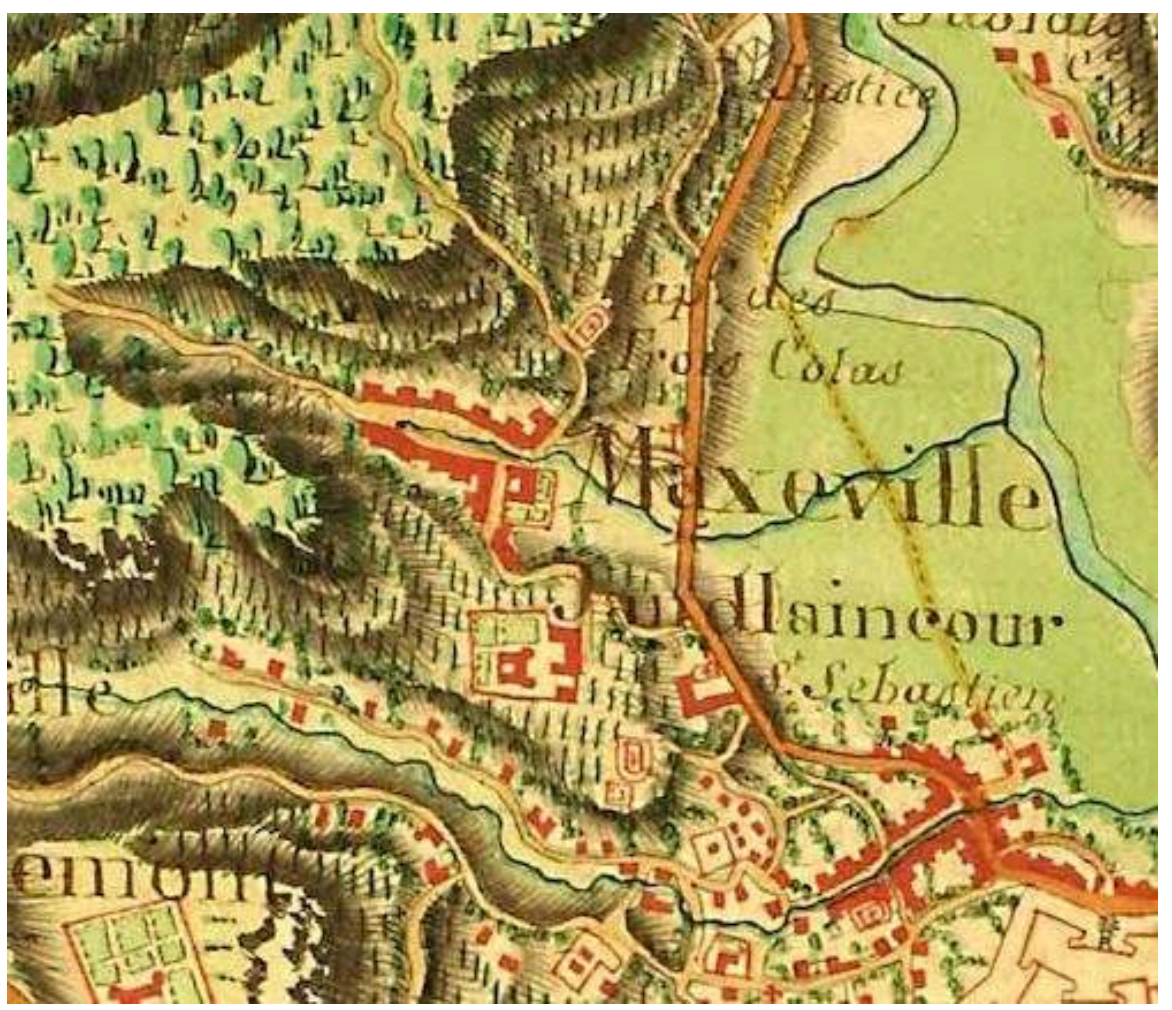

Au nord-ouest de Nancy dont on découvre les fortifications rasantes (en bas, à gauche de la figure). Le talus est drapé de vignes tout autour du village-rue de Maxéville logé dans l'incision faite par un ruisseau cataclinal qui dévale jusqu'à la Meurthe, avec un lit majeur occupé par des prairies humides. À l'exception de certains sites trop pentus, marqués par des affaissements miniers à associer à l'ancienne exploitation de la couche de minerai de fer ou encore sur le mince bandeau des argiles à gonflement qui se situent sur le pied de cote, tout est construit.

Les retrouvailles d'une collectivité avec des espaces oubliés et marginalisés s'opèrent sur au moins trois niveaux. Elles concernent d'abord le groupe de parcelles identifiées par un toponyme qu'il a souvent fallu restaurer, ce qui est facilité si le nom est évocateur: Mi-les-Vignes, les Rachouts, etc. Le lieu qui retrouve son nom correspond aussi à un zonage du PLU, cadré sur la matrice cadastrale, avec la prise en compte des éventuelles contraintes apposées. Ensuite s'exprime la mise en linéaire, continuité et connexité des corridors dessinés. Enfin, très souvent, la question des vignobles reliques, oubliés, enfrichés, est associée à l'entretien des lisières forestières dont l'apparence, la pérennité et l'organisation varient beaucoup dans le temps et l'espace en fonction de la nature et de la taille de la propriété. Sur l'agglomération de Nancy, le classement en cours du massif de Haye en forêt de protection renouvelle cette question puisque le bandeau, sur lequel sera définie la limite, correspond en grande partie au talus bien exposé au regard depuis la ville. Ici se sont exprimées des dynamiques inverses d'extension puis de recul des vignes, des vergers, des friches et des processus de reconquêtes forestières associés à des formes de mitage (Husson, 2009). C'est à partir du télescopage de ces trois échelles que peut se dessiner une politique globale, systémique, prudente de remise en ordre et en phase entre les actuels besoins des espaces évoqués et le reste de la ville. Par le passé, les vignes accolées à la cité ont été des lieux de labeur, avec très souvent la mobilisation d'une débauche de travail pour dominer des espaces ingrats. Il fallait épierrer, corriger les pentes, ériger des murets, etc. Dans la 
filiation paysage-société, après avoir été craints, plus ou moins abandonnés, ces lieux réapprennent à vivre en aménité. On reconnaît la dimension aimable de leurs chemins bordés de trognes, avec du plessage (branches souples vivantes ou coupées tressées) et des églantiers, parfois la rencontre d'un hérisson ou d'un écureuil. Le paysagepalimpseste des vignes a été modelé par des usages anciens mais revit, retrouve du sens et se réinsère dans des connivences nouvelles faites avec les habitants et les usager des lieux. Ces espaces forment des bornes ou, à plus juste façon de parler, des îlots de nature dans la ville et sur ses marges. Il s'agit de lieux de respiration, d'habitats, de refuges et encore de relatif silence; des zones qui tamponnent les bruits. Les choix de ménagement des sites sont effectués pour obéir au devoir de transmettre et si possible d'aggrader le legs. C'est là le principe élémentaire de toute politique soutenable. Ces choix amènent à articuler le respect des héritages légués par le temps qui passe (en particulier le passage depuis l'aube du XVIII siècle de l'échelle de la cité enmuraillée à la ville puis à l'actuelle urbanisation étalée, l'« urbain sans figure " déploré par Michel Lussault), avec les directives actuelles appliquées au foncier et encore le modelage des formes paysagères capables d'évoluer dans des arbitrages satisfaisants. Sur le pourtour de l'agglomération de Nancy, l'inventaire de la ressource, la prise en compte de la taille et de la nature juridique de la propriété (État, collectivités, autres personnes morales, particuliers, parcelles en déshérence), le relevé du type d'occupation des sols sont, comme ailleurs, le préalable à l'action faite d'arbitrages entre ce qui sera construit, transformé en infrastructure (pénétrantes urbaines, cimetière) ou conservé en nature. Pour une infime partie, on a rétabli des vignes $\left(347 \mathrm{~m}^{2}\right.$ à Laxou dès 2000, 1100 pieds d'Auxerois et 180 pieds de cépage Régent offerts, dans le cadre d'un jumelage francoallemand plantés, sur $3000 \mathrm{~m}^{2}$ à Dommartemont en 2003).

8 Pour le reste, il s'agit d'inventer des gestions différentiées de ces espaces verts originaux dont l'intérêt est reconnu par l'Agenda 21 local qui associe une façon de penser globale et écosystémique à une action locale. Il s'agit de friches parfois inaccessibles, hirsutes, maintenues en l'état sur un sol mince ou évoluant en une formation arborée dans d'autres cas. On trouve aussi des jardins privés ou loués par les communes ou des associations, et encore des vergers, petits prés. Des pelouses sèches dominent, situées entre les anciennes vignes et les lisières, sur le haut des talus et le début du revers de côte. Ces espaces sensibles, précieux, parfois classés en ENS ${ }^{2}$, ont souvent été préservés grâce à la présence tardive des militaires qui, pour les exercices de manœuvres, avaient conservé ces espaces ouverts. Lisières, pelouses sèches, vergers, vignes, jardins dessinent le cadre agreste local. La présence des éléments cités est à géométrie variable. Pour faire perdurer une bonne cohérence écosystémique de l'ensemble, satisfaire nos actuels besoins, répondre aux demandes sociales exprimées, donner aux paysages visibles une bonne lisibilité, il est nécessaire de bien articuler les échelles des objets évoqués. Or, celles-ci changent, évoluent dans leurs contenus. Les actuels projets de ville invitent à la fois à densifier le tissu urbain et à augmenter la cohérence écologique au sein du tissu urbain. L'harmonisation de ces deux objectifs ne va pas sans friction et difficulté. Le $\operatorname{Scot}^{3}$ préconise d'économiser l'espace et de préserver la biodiversité en application de la loi du Grenelle 2 de juillet 2010. Le PLU ${ }^{4}$ identifie désormais les trames vertes et bleues avec, au sein du PADD ${ }^{5}$, l'affichage de la lutte contre l'étalement. Tout à la fois, il faut tenter de concilier ce qui peut être délicat à l'être : densifier et conserver de l'espace nature et de l'interstitiel agricole. Dans ce contexte, le principal levier semble être le $\cos ^{6}$ associé à la conduite d'un $\mathrm{PLH}^{7}$ qui accorde la priorité à trois directives : la redensification du tissu urbain, la mixité aidée 
par la diversification choisie par la variété des tailles de logements, enfin la construction économe.

Pour éviter les psychosystèmes, les lieux noués où se focalisent des tensions fortes, des oppositions, des replis arc-boutés et fermés à la négociation, il est important de communiquer sur les lieux, de les arrimer à plusieurs valeurs afin de construire et de faire évoluer un projet. Il s'agit en particulier de la narration et de l'artialisation apportée aux lieux.

\section{Renaître, célébrer, transmettre : comment ? pour qui ? pour quelles attentes?}

10 La nature urbaine relictuelle est dans la catégorie des tiers espaces (Novarina, 2003). Elle entre en force dans les interrogations portées par l'aménagement, avec en particulier la prise en compte des trames dans les PLU. Cet objectif est à rapprocher des biais retenus pour densifier les bâtis, en finir avec les scénarios permissifs, exclusifs qui encourageaient de faibles COS, avec pour corrélat l'étalement de la ville. Dorénavant, l'objectif affiché est de redéfinir un couple densité/diversité créateur d'émulation. Ainsi, les tiers espaces évoqués rentrent en force dans nos préoccupations d'aménagement/aménagement de l'espace. Ils revêtent et portent plusieurs enjeux. Les paysages ordinaires appartiennent à nos ressentis et participent aux connivences que nous entretenons avec les îlots tracés par des chemins, des jardins, des friches, des reliquats de vignes qui ont été épargnés. Le succès de fréquentation de ces lieux remis en valeur et sécurisés va grandissant. Par ailleurs, au sein des PLU, ces sites sont aussi les territoires de toutes les querelles et ambitions, en particulier s'il y a du terrain bâtissable ou, pire encore, des communes qui disposent encore de foncier mais n'ont pas atteint leur quota de logement social. Bref, les territoires évoqués ont au moins quatre lectures possibles : poétique, écologique, sociale, enfin économique. Ces quatre approches cohabitent mal et sont porteuses de tension. Le recours à la fête (Di Méo, 2001), la mise en événement (Jacopin, Husson, 2008) et l'appui aux références historiques qui apportent de la respectabilité aux lieux et de la patine aux paysages sont d'excellents moyens pour éclairer ces sites, en souligner la valeur et faire lever le levain de la gouvernance. Les élus ne s'y sont pas trompés, orchestrent ces mises en représentation (Debarbieux, 1995) de ressources territoriales locales parfois en cours de patrimonialisation (Veschambre, 2007; Andrieux, 1997), faisant l'alchimie entre les successions de temps (ici, la succession entre la vigne, les vergers, les friches, l'actuelle renaissance), les espaces (les îlots, corridors, trames vertes) et les formes (parcellaires hérités, figés, regroupés). 
Figure 2. Petite parcelle de cinq ares de jardin située à Laxou-Mi-les-Vignes

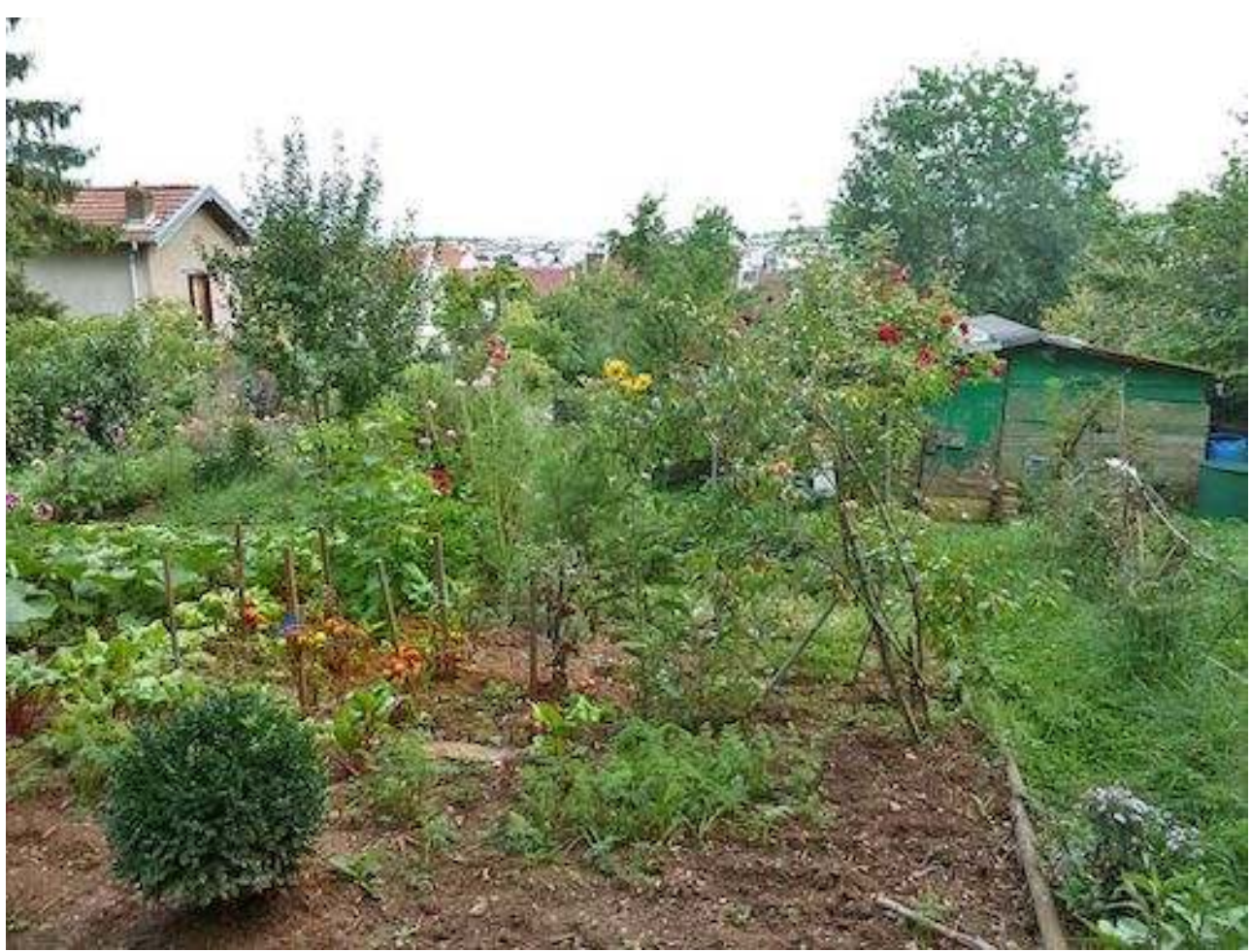

Dessinée en L, piquetée de ceps et de pêchers de vigne, close par une haie biodiversifiée, avec des dalles de calcaire dressées de champ, et de temps immémorial, pour freiner la descente du sol. Bien protégée car placée au pied du talus, la parcelle est sur un sol léger, assez pierreux et qui se réchauffe vite. On est ici dans un environnement agreste pourtant placé à moins de deux kilomètres du centreville. Des parcelles voisines sont en vignes, en vergers, en friches arbustives et en bosquets de frênes et d'érables.

Trois approches sont privilégiées pour entrer et conforter cette renaissance portée par la sympathie et l'adhésion des citoyens qui s'impliquent assez bien dans les processus de gouvernance et de pratiques associatives sur ces sujets.

D'abord, vignes et reliquats d'espaces naturels méritent d'être intégrés dans des OPAV (opérations programmées d'amélioration des vergers) en ayant soin de faire renaître des microtoponymes pleins de saveurs; bref de renommer des lieux qui étaient entrés dans l'anonymat. À nouveau, d'étroits chemins sont rendus à la circulation piétonne, fléchés, odonymés, entretenus, régulièrement fauchés (Sahuc, 2010). Dans ce contexte de réappropriation des lieux, réapparaissent des lambeaux de vigne, des treilles, voire des carrés de vigne plantés à l'initiative des communes, d'associations ou de particuliers. Ces formes de restauration fonctionnent dans des échelles emboîtées de solidarité écologique, d'abord au niveau de la réunion de parcelles défrichées, dégagées, remises en culture (avec l'inconvénient de voir prospérer des linéaires de grillages si des haies variées ne sont pas parallèlement mises en place, avec des frênes, des noisetiers, des buissons ardents et d'autres arbustes se prêtant bien à la taille mais encore des mûriers, des églantiers, etc.), ensuite, dans la mise en scène des lieux, en intégrant un plan-paysage mis en cohérence avec le cadrage d'un Agenda 21 local, ce qui peut être traduit par des cartes d'exposition au regard particulièrement utiles sur les sites en cuvette, pentus (c'est le cas des villes du sillon lorrain logées au pied des systèmes de côte). 

lumière par l'organisation d'événements festifs, ludiques, avec pour prétexte l'appui de récits paysagers. C'est par exemple « la mise en conte» des chemins relus et revus par le récit d'une histoire. Celle-ci invite à être en retrouvaille avec un lieu, voire à donner du goût à l'espace réinvesti, grâce à l'articulation du réel et de l'imaginaire. L'empathie, ici l'identification affective à un lieu, est un point de départ pour améliorer la représentation des espaces qui, en analyse géohistorique, ajoute aux mosaïques et aux diverticules des phénomènes de gommage, de concurrence, de superposition. Le récit invite à rêver sur tout cela. L'accompagner d'un goûter, d'un jeu de piste ou autre activité récréative conforte les efforts de reconnaissance entrepris. Ces démarches intègrent des effets de mode et sont consensuelles, créent des envies de pseudocampagne dans la ville, avec des espaces réaménagés pour séduire, repris par le goût de vivre (Husson, 2011). Ici sont mobilisés tous les sens, ce qui avait déjà été avancé par Charles Avocat (1982) quand il établissait ses grilles de lecture sensible des paysages. La fête est une façon agréable d'ausculter le territoire. Elle associe le terrain et l'imaginaire qui apporte un supplément de sens aux lieux. Plus prosaïquement, c'est un levier pour consommer à nouveau un espace qui avait été écarté, oublié, marginalisé et le faire émerger par le haut.

Le projet de retrouvaille et de renaissance d'un territoire invite à le replacer dans un environnement élargi. Pour beaucoup, les vignobles septentrionaux ont grandi à portée des villes et des monastères. Très majoritairement, ils ont recherché la proximité des lisières forestières qui réduisaient les chocs thermiques, tamponnaient les effets des grêles et réduisaient l'impétuosité des vents. Les lisières héliophiles fournissaient en abondance des échalas taillés dans de jeunes robiniers. Aujourd'hui, la renaissance des tiers espaces en vignes, en vergers et en friches est à associer au flux, au reflux ou encore à la stabilité des lisières forestières périurbaines. À Nancy, le classement en cours des 15000 ha du massif de Haye en forêt de protection est un avantage pour concevoir une approche durable des trames vertes qui s'inscrivent dans ce continuum.

Investir la reconnaissance des anciens vignobles enkystés, digérés, dégradés ou en renaissance dans l'actuel tissu urbain dilaté et étalé est une thématique originale. Pour aboutir, cette contribution à l'écologie urbaine impose la transdisciplinarité, partant de l'inventaire et de l'histoire de la ressource foncière pour se conclure avec l'aménagement et la publicisation par la mise en événement des lieux retrouvés, reconnus, remis en valeur. Actuellement, ces envies qui intègrent à la fois tensions, conflits, efforts de gouvernance et poésie dans les projets entrepris font de ces lieux réappropriés des pièces importantes des trames vertes et bleues dans les villes.

\section{BIBLIOGRAPHIE}

Andrieux, J.-Y., Patrimoine et histoire, Paris, Belin, 1997, 283 p.

Arnaud, J.-L., Analyse spatiale, cartographie et histoire urbaine, Paris, Parenthèse/MMSH, 2008, 233 p. 
Avocat, C., « Approche du paysage », Revue de géographie de Lyon, $\mathrm{n}^{\circ} 4,1982$, p. 333-342.

Bertrand, $\mathrm{Cl}$. et $\mathrm{G}$., Une géographie traversière, l'environnement à travers territoires et temporalités, Paris, Arguments, 311 p.

Debarbieux, B. « Le lieu, le territoire et trois figures de rhétorique »., L'Espace géographique, $\mathrm{n}^{\circ} 2$, Paris, 1995, p. 97-112.

Di Méo, G. (textes réunis), La Géographie en fête, Gap, Géophrys, 2001, 265 p.

Dion, R., Le Paysage et la vigne. Essai de géographie historique, Paris, Payot, 1990, 294 p.

Girel, J. «Quand le passé éclaire le présent : écologie et histoire du paysage », Géocarrefour, Lyon, $\mathrm{n}^{\circ} 4,2006$, p. 249-264.

Godet, L. « La "nature ordinaire" dans le monde occidental », Espace géographique, n 4 , Paris, 2010, p. 295-308.

Husson, J.-P., « La vigne dans l'agglomération de Nancy, de l'objet relique au projet de renaturation », Revue géographique de l'Est, $\mathrm{n}^{\circ}$ 1-2, Nancy, 2004, p. 81-87, URL : https:// journals.openedition.org/rge/1057.

Husson, J.-P., « Le classement en forêt de protection du massif de Haye », Nancy, Revue géographique de l'Est, ${ }^{\text {os }}$ 2-3, Nancy, 2009, URL : http://rge.revues.org/1872.

Husson, J.-P., « Le Paysage révélé par l'événement festif : pourquoi ? comment ? avec quels résultats? ", Comité des Travaux historiques et scientifiques, Neufchâtel, 2011, sous presse.

Jacopin, R., Husson, J.-P., Benoit, M. « La fête du paysage, objet de réflexion sur les territoires » dans Appel, V. et al., La Mise en culture des territoires, nouvelles formes de cultures événementielles et initiatives des collectivités territoriales, Nancy, PUN, 2008, p. 53-64, 275 p.

Lachiver, M., Vins, Vignes et Vignerons, Paris, Fayard, 1997, 724 p.

Lepetit, B. « Histoire urbaine et espace », L’Espace géographique, n 1, Paris, 1980, p. 43-54.

Lussault, M., L'Homme spatial, Paris, Seuil, 2007, p. 273, 364 p.

Martin, J.-Cl., " La création de paysages viticoles : une histoire de matériaux », Sud-ouest européen, $\mathrm{n}^{\circ} 21$, Toulouse, 2006, p. 29-35.

Novarina, G. « Ville diffuse et système vert », Revue de géographie alpine, n 4, 2003, Grenoble, p. 9-17.

Plet, F., « Vignobles et vignes : les formes nouvelles d'une vieille liaison », Cahiers nantais, $\mathrm{n}^{\circ} 58$, Nantes, 2002, p. 111-126.

Prêcheur, $\mathrm{Cl}$., « Nancy. Rapports de l'actuelle structure urbaine et de l'ancienne structure agraire », B.A.G.F., n 235-236, Paris, 1953, p. 106-115.

Robert, S. «Comment les formes du passé se transmettent-elles? », Études rurales, $\mathrm{n}^{\circ}$ 167-168, Paris, 2003, p. 115-132.

Roncayolo, M., Les Grammaires d'une ville. Essai sur la genèse des structures urbaines à Marseille, Paris, EHESS, 1996, $607 \mathrm{p}$.

Roupnel, G., Histoire de la campagne française, Paris, Club des libraires de France, 1955, 313 p.

Sahuc, P., «Chemins périurbains : aménités vécues et enjeux réels », Revue Nature Sciences et Sociétés, $\mathrm{n}^{\circ} 2$, Paris, 2010, p. 147-157. 
Velascot-Graciet, H. (travaux réunis), Territoires, Mobilités et Sociétés, Bordeaux, Maison des sciences de l'homme d'Aquitaine, 2009, 348 p.

Veschambre, V. « Patrimoine, un objet révélateur des évolutions de la géographie et de sa place dans les sciences sociales ", Annales de géographie, n 4, Paris, 2007.

\section{NOTES}

1. LIDAR : Light Detection And Ranging.

2. ENS : Espace naturel sensible.

3. Scot : Schéma de cohérence territoriale.

4. PLU : Plan local d'urbanisme.

5. PADD : Plan d'aménagement et de développement durable.

6. COS : Coefficient d'occupation du sol.

7. PLH : Plan local d'habitat.

\section{RÉSUMÉS}

Les villes anciennes étaient entourées de petites parcelles de vignes, de vergers, de jardins en alternance avec de vastes espaces: des prairies inondables et des grandes propriétés. Partiellement préservées par l'étalement urbain, ces bornes à la ville renaissent après avoir été oubliées. Les objets qui les composent accèdent désormais au statut de réserve de biodiversité, s'inscrivent dans des trames vertes et bleues connexes entre elles. Ces lieux deviennent patrimoine, mémoire et s'intègrent dans des projets de paysages tissés entre écologie, histoire et ménagement des territoires. De nouveau aménagés, ils sont reconnus par les leviers que sont l'événement festif et l'entrée en gouvernance. L'étude de cas appliquée au Grand Nancy illustre ce propos.

Cities used to be sourrounded by small tracts of vineyards, orchards, garden, in addition to vast estates and meadows. This landscape environment was partly overlaid by urban sprawl but sometimes goes through a kind of renaissance. It is now contemplated as part of the urban green infrastructure and urban cultural heritage. It can be accounted for again through a new city governance.

\section{INDEX}

Mots-clés : vignes, ville, image, connexité, trame, Nancy

Keywords : vineyard, city, image, green infrastructure, Nancy 
AUTEUR

JEAN-PIERRE HUSSON

Professeur de géographie à Nancy 2. Membre du Centre d'études et de recherche sur le paysages (Cerpa), EA 1135.

Jean-Pierre.Husson[at]univ-nancy2[dot]fr 